\section{Spül-Drainage und Fibrinolyse zur Behandlung des metapneumonischen Pleuraempyems*}

\author{
D. J. M. Frey ${ }^{1}$, J. Klapa², D. Kaiser ${ }^{2}$ \\ ${ }^{1} \mathrm{G}$. ö. Krankenhausgesellschaft Ibbenbüren mbH, Abt. Thorax- \\ und Kardiovascularchirurgie, Ibbenbüren \\ 2 Lungenklinik Heckeshorn, Abteilung Thoraxchirurgie, \\ Krankenhaus Zehlendorf, Berlin
}

Zusammenfassung: Es wird über die Behandlung von $336 \mathrm{~Pa}$ tienten (Pat.) mit manifestem Pleuraempyem in einem Zeitraum von 10 Jahren (1985-1995) berichtet. Bezogen auf die Genese der Erkrankung werden besonders die Behandlungsergebnisse von 218 Pat. mit „metapneumonischem Pleuraempyem“ retrospektiv analysiert. Dabei konnten 201 Pat. $(=92,2 \%)$ erfolgreich mit Spül-Drainage therapiert werden. Nur in 11 Fällen waren chirurgische Eingriffe nötig (=5\%). Ungeheilt blieben 6 Pat.: Einmal wurde palliativ eine Dauerdrainage eingelegt und 5 Pat. verstarben im Laufe der Behandlung (Mortalität $=2,3 \%$ ). Die Spülbehandlung wurde in den ersten Jahren mit einfachem Drain $(n=38)$ bzw. ab 1989 als DoppellumenDrainage $(n=158)$, seit 1987 in den meisten Fällen mit intrapleuraler, medikamentöser Fibrinolyse - im Mittel an 12,96 Tagen - durch Instillation von Varidase $N^{\circledR}(n=182)$ durchgeführt. Bei separater Betrachtung auswärts invasiv vorbehandelter Patienten $(n=30)$ zeigte sich eine statistische Abhängigkeit zwischen Behandlungszeiten und Art der Behandlung: Ohne signifikanten Unterschied zwischen den Gruppen (Gr) lag die mittlere Behandlungszeit bei Anwendung eines Drains ohne Fibrinolyse (Gr1) bei 31,8 Tagen, bei einem Drain mit Fibrinolyse (Gr2) bei 26,5 Tagen. Eine deutliche Verkürzung der Behandlungsdauer wird bei Anwendung der Fibrinolyse in Kombination mit einem Doppellumen-System beobachtet: Bei 2 Drains $(G r 4)$ auf 20,6 Tage und bei doppellumigem Einzeldrain (Gr5) auf 19,8 Tage mit Signifikanznachweis im Vergleich der Gr4 mit Gr1 $(p=0,005)$, der Gr5 mit Gr1 ( $p<0,001)$ und der Gr5 mit Gr2 $(p=0,014)$. Eine signifikant längere Behandlungsdauer ( $p<0,001$ ) weist mit 40,6 Tagen die Gruppe der vorbehandelten Patienten im Vergleich mit den jeweils korrespondierenden Gruppen Gr4 bzw. Gr5 auf. Es wird gefolgert: Metapneumonische Empyeme lassen sich in weitaus den meisten Fällen durch Spüldrainage ausheilen. Die Mortalität ist vergleichsweise gering. Bei Kombination eines Doppellumen-Drains mit intrapleuraler Instillation eines fibrinolytischen Medikamentes (Varidase $N^{\circledR}$ ) ergibt sich die kürzeste Behandlungsdauer. Invasiv vorbehandelte Patienten benötigen bei gleicher Behandlungsart signifikant längere Behandlungszeiten.

Irrigation Drainage and Fibrinolysis for Treatment of Parapneumonic Pleural Empyema: We report on our experiences on 336 patients suffering from manifest pleural empyema within a period of 10 years (1985 - 1995). Considering the

Pneumologie 53 (1999) 596-604

(c) Georg Thieme Verlag Stuttgart · New York pathogenesis, particularly the results of 218 patients with „parapneumonic pleural empyema“" were analysed retrospectively. Definite healing could be achieved by chest tube placement and pleural irrigation in 201 patients (=92,2\%). Other 11 patients finally needed surgical interventions $(=5 \%)$. Only 6 patients could not be cured: An indwelling tube was palliatively inserted once and 5 patients died in the course of the medical treatment (mortality $=2.3 \%$ ). Within the first years the irrigation therapy was performed using a single chest tube ( $\mathrm{n}=$ $38 \%$ ) but since 1989 a double-lumen drainage was used ( $\mathrm{n}=$ 158). Since 1987 in most cases $(n=182)$ intrapleural medicinal fibrinolysis was performed by instillation of streptokinase (Varidase $\left.N^{\circledR}\right)$. If outward invasively pretreated patients $(n=30)$ are analysed separately, a statistical dependency can be found between the duration and the way of treatment. Without significant difference between the groups $(G r)$ the average duration of treatment using a single tube without fibrinolysis (Gr1) was 31.8 days, but 26.5 days using a single tube combined with fibrinolysis (Gr2). A clear shortening of the duration is detectable if patients were treated with a combination of double lumen drainage and fibrinolysis: If 2 tubes were used $(\mathrm{Gr} 4)$ the treatment lasted 20.6 days, using one double-lumen tube (Gr5) it took 19.8 days. There is a proof of significance at comparison of Gr4 with $G r 1$ ( $p=0.005)$, Gr5 with Gr1 ( $p<0.001)$ and Gr5 with $\mathrm{Gr} 2(\mathrm{p}=0.014)$ respectively. A significant longer duration of treatment ( 40.6 days, $p<0.001$ ) is found for the group of the pretreated patients, if compared with the corresponding groups Gr4 or Gr5. Conclusion: Parapneumonic empyemas most often can be cured by irrigation drainage. The mortality is comparatively low. The shortest duration of treatment is needed using the combination of a double-lumen tube with intrapleural instillation of a fibrinolytic agent (Varidase $\mathrm{N}^{\circledR}$ ). Invasively pretreated patients need significantly longer durations at same form of treatment.

Das Pleuraempyem ist seit Einführung der Antibiotika eine zwar seltene, jedoch immer noch folgenschwere Erkrankung. Im Krankengut unserer spezialisierten Lungenklinik mit Thoraxchirurgie mit überregionalem, großstädtischem Einzugsgebiet in Berlin wurden in einem Zeitraum von 10 Jahren (19851995) insgesamt 336 Patienten (Pat.) mit Empyemen unterschiedlichster Ursache behandelt. Dies entspricht einer Häu-

\footnotetext{
* Wesentliche Teile dieser Arbeit sind der Dissertation von Herrn Jan
} Klapa aus der Lungenklinik Heckeshorn entnommen. 
figkeit von ca. 0,6\% am gesamten stationären Patientengut dieser Klinik. Die Häufigkeit dieser Erkrankung innerhalb der Bevölkerung ist unbekannt. Bei Berücksichtigung der Zuweisungs-Praxis erscheint uns eine Häufigkeit von maximal 50 Fällen pro 1 Mill. Einwohner/Jahr $(=1 / 20000)$ realistisch. Dies bedeutet, daß auch in großen Kliniken kaum mehr als 5-10 Fälle pro Jahr diagnostiziert werden, so daß eine fundierte eigene Erfahrung kaum gewonnen werden kann. Dieses ist jedoch für die Behandlung dieser Erkrankung wie auch für die Wertung der in der Fachliteratur propagierten, ganz unterschiedlichen Behandlungsstrategien unverzichtbar.

Auch in der neueren Literatur werden noch hohe Letalitätsraten angegeben (z.B. maximal $58 \%$ bei konservativer, $16 \%$ bei operativer Behandlung [31] bzw. 17,6\% in einer Multizenterstudie bei verschiedenen konservativen und operativen Behandlungsregimen ( $n=119$ Pat.) [8], die denjenigen früherer Jahre - aus den Jahren 1980-1991 wird eine maximale Letalität von $53 \%$ zitiert [33] - durchaus entsprechen. Dies wird darauf zurückgeführt, daß ein Pleuraempyem sich überwiegend bei Pat. mit geschwächter Körperabwehr, z.B. als erschwerende Komplikation einer anderen Grunderkrankung, bei Alkoholabusus oder bei alten, hinfälligen Pat. entwickelt. Derartige Ergebnisse sollten jedoch nicht mehr akzeptiert werden, da durch optimierte Therapiekonzepte auch bei ähnlich kompliziertem Krankengut eine deutlich geringere Sterblichkeit erreicht werden kann.

Für die Empyembehandlung werden - seit Jahrzehnten unverändert - mehrere Verfahren beschrieben: Die gelegentlich noch erwähnte Punktion gilt heute jedoch allgemein als obsolet, sie ist nur als diagnostische Maßnahme indiziert und hier unverzichtbar. Häufig wird die Drainagebehandlung mit einer regelmäßigen Spülung der Thoraxhöhle kombiniert. Der „offenen Drainage“ kann eine Gruppe von Verfahren zugeordnet werden, bei denen tatsächlich ein mechanisches Debridement der Thoraxhöhle mit anschließender konventioneller Drainagebehandlung ausgeführt wird. Hierzu gehören sowohl konventionell-thorakoskopische Verfahren (u.a. mit dem Mediastinoskop) bzw. die neuerlich propagierten videoassistierten thoraxchirurgischen Operationen (MIC/VATS) als auch - in irreführender Nomenklatur - die „Früh-Dekortikation“. Bei einer Empyemektomie ist obligat eine Dekortikation auszuführen mit Entfernung der Schwiele und beider Pleurablätter. Thoraxfenster und Thorakoplastik wurden früher besonders bei Tuberkulose-Erkrankungen angewendet; sie stellen allerdings auch heute noch in Einzelfällen wertvolle Behandlungsoptionen dar.

Mit der Entwicklung und Verwendung der Antibiotika konnten erstmals wesentliche Prognose-Verbesserungen erzielt, keinesfalls jedoch die invasiven Maßnahmen ersetzt werden. Die intrathorakale, medikamentöse Fibrinolyse - 1949 erstmalig [38] und in den 50er Jahren häufiger beschrieben erlebt in den letzten Jahren in der Literatur eine „Renaissance“. Die Wirkungsweise ist bekannt und schon 1952 ausführlich dargestellt [13]. Durch Kombination der Fibrinolyse mit einer „doppelläufigen“ Spüldrainage läßt sich - nach eigener Erfahrung [10] - in den meisten, auch komplizierten Fällen eine Heilung erreichen.

Angaben zu Behandlungsergebnissen in der Literatur spiegeln meist die bevorzugten Verfahren in der jeweiligen Institution

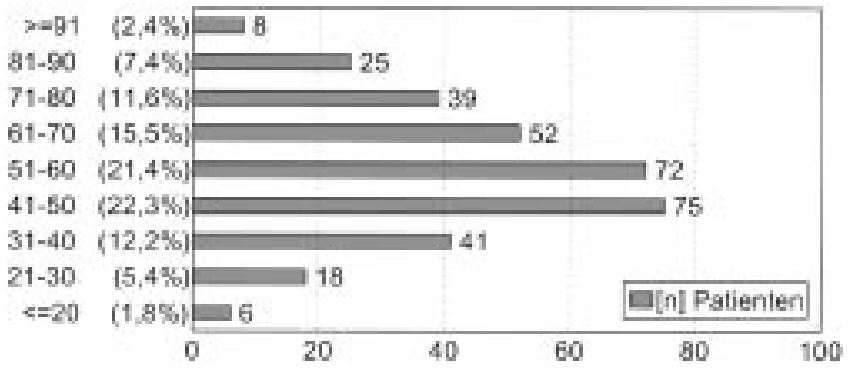

Abb. 1 Pleuraempyem. Altersverteilung der Patienten $(n=336)$.

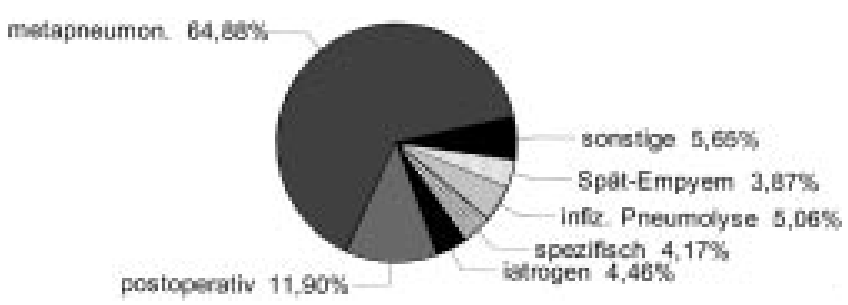

Abb. 2 Pleuraempyem. Genese $(n=336)$.

wider, so daß sich hieraus keinerlei „Standard“ ableiten läßt. So zeigte eine Befragung von Teilnehmern $(n=242)$ der „1991 American College of Chest Physicians Annual Scientific Assembly“ unterschiedliche Behandlungspräferenzen beim vielkammerigen Pleuraempyem (49\% Pleura-Dekortikation; 22\% „Offene“ Pleuradrainge; 14\% Pleuradrains \& Fibrinolyse; 8\% einfache Pleuradrainage; $7 \%$ CT-gesteuerte Einlage mehrerer kleinlumiger Drains) [34]. Bei erkennbarer Bevorzugung operativer Maßnahmen werden diese oft initial oder nach allenfalls kurzzeitiger und kaum optimaler Anwendung konservativer Drainageverfahren - und damit zwangsläufig „unbefriedigendem“"Ergebnis - eingesetzt. Ohne Berücksichtigung von Risiken und Nebeneffekten chirurgischer Methoden oder postoperativer Morbidität werden häufig nur pauschale „Heilungs“-Quoten genannt und dabei als Vorteil eine „kurze“ stationäre Behandlungsdauer angeführt.

Wenn aber „Heilung“ auf verschiedenen Wegen erreicht werden kann, müssen notwendigerweise die jeweiligen Risiken wie auch die Belastung der Patienten durch die Behandlung oder deren Folgen berücksichtigt werden. Bei ökonomischen Vergleichen sind neben den Kosten einer

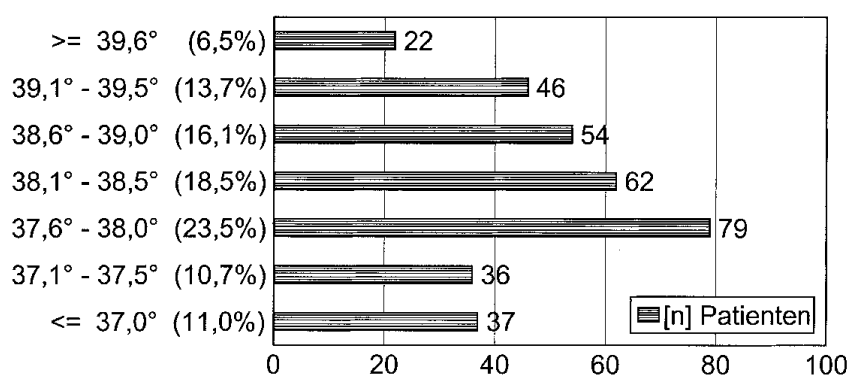

Abb. 3 Pleuraemypem. Körpertemperatur bei Behandlungsbeginn 


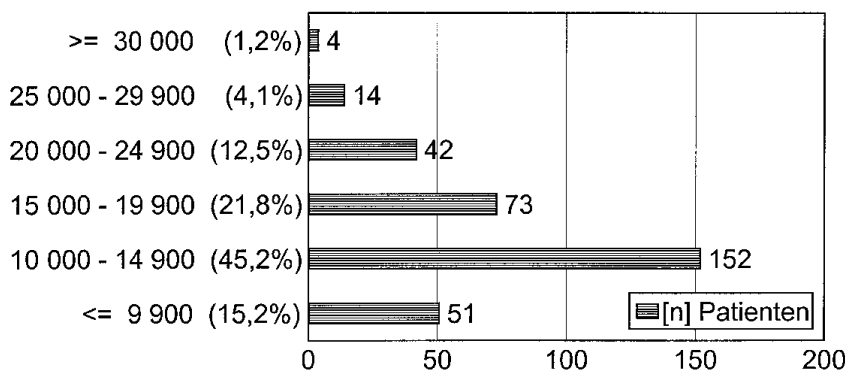

Abb. 4 Pleuraempyem. Leukozytenzahl bei Behandlungsbeginn.

Behandlung selbst auch Kosten typischer Folgen und Komplikationen bis hin zu Transfusions-Risiken (Hepatitis-/HIVInfektionen) zu berücksichtigen.

In der vorliegenden Arbeit sollen unsere Erfahrungen an einem vergleichsweise großen Krankengut aus überschaubarem Zeitraum dargestellt werden. Die konsequente Ausschöpfung konservativer Möglichkeiten ermöglicht eine fundierte Aussage hinsichtlich erreichbarer Ergebnisse. Besonderes Berücksichtigung soll die größte und relativ homogene Gruppe „metapneumonischer Empyeme“ finden, da Empyeme anderer Genese vorzugsweise im Krankengut spezialisierter Zentren auftreten und - nach unserer Erfahrung - immer eine differenzierte und individuelle Behandlung erfordern.

\section{Krankengut}

In einem Zeitraum von 10 Jahren (1985 - 1995) wurden in der Abt. Thoraxchirurgie der Lungenklinik Heckeshorn in Berlin 336 Pat. (244 männlich $=72,6 \%$, 92 weiblich $=27,4 \%$ ) mit einem manifesten Pleuraempyem - im Stadium II (fibrinopurulente Phase) oder Stadium III (Organisationsphase) behandelt. Die Altersverteilung ist in Abb.1 dargestellt. Bei minimal 16 und maximal 92 Jahren lag das Durchschnittsalter bei 52,7 Jahren mit einer Häufung im mittleren Lebensalter. Bezogen auf die Genese lassen sich 7 Hauptgruppen bilden (Abb.2), wobei die Gruppe der metapneumonischen Empyeme mit 218 Pat. weitaus die größte darstellt. Postoperative Empyeme $(n=40)$ traten bei 3909 konsekutiven Thorakotomien mit einer Inzidenz von 1,02\% auf, 31 mal nach aseptischelektiven und 9mal nach primär septischen Operationen. Als Spätempyeme $(n=13)$ wurden Fälle klassifiziert, die mehr als 6 Monate postoperativ überwiegend als Folge einer Bronchusstumpf-Spät-Insuffizienz nach Pneumektomie auftraten. Pneumolysen-Empyeme $(\mathrm{n}=17)$ sind - als Spätkomplikation durch Tuberkulose-Exazerbation und/oder unspezifische Superinfektion in einem Abstand von 30 bis 40 Jahren nach Pneumolyse auftretend - zwar selten aber typisch, und führen gelegentlich zum hier ebenfalls enthaltenen Empyema necessitatis. Dagegen handelt es sich bei den „spezifischen“ Empyemen $(n=14)$ um frische pleurale Tuberkulose-Erkrankungen mit Keimnachweis. Als „iatrogenes“ Empyem $(\mathrm{n}=15)$ wurde die Infektion eines ursprünglich sterilen Pleuraergusses - meist maligner Ursache - durch diagnostische oder teilweise mehrfache therapeutische Punktionen, Drainagebehandlungen oder Thorakoskopien bezeichnet.

Klinisch bestand initial nur in etwa einem Drittel der Fälle eindeutiges Fieber $>38,5^{\circ} \mathrm{C}$ (siehe Abb. 3), jedoch bei ca. $85 \%$
Tab. 1 Pleuraempyeme $(n=336)$. Behandlungsergebnisse bei Differenzierung nach Genese und Behandlungsverfahren. (Angegeben ist die zur Heilung führende Behandlungsart bzw. der Anteil palliativ mit Dauer-Drainage versorgten und der verstorbenen Patienten)

Drainage Dekorti- Thorax- Dauer- Tod Summe kation fenster Drain

\begin{tabular}{lrrrrrr}
\hline metapneumonisch & 201 & 9 & 2 & 1 & 5 & 218 \\
postoperativ & 22 & - & 16 & - & 2 & 40 \\
Pneumolyse & - & - & 17 & - & - & 17 \\
iatrogen & 11 & - & - & 2 & 2 & 15 \\
spezifisch (Tbc) & 9 & 3 & - & - & 2 & 14 \\
Spätempyem & 2 & - & 11 & - & - & 13 \\
sonstige & 12 & 1 & - & 2 & 4 & 19 \\
Summe & 257 & 13 & 46 & 5 & 15 & 336 \\
\hline
\end{tabular}

eine - vorwiegend mäßige - Leukozytose (siehe Abb.4). Das Empyem wurde normalerweise durch Probepunktion mit Aspiration von eindeutig eitrigem Pleurasekret diagnostiziert; das gewonnene Material wurde grundsätzlilch bakteriologisch untersucht. In 303 von 336 Proben $(n=90,2 \%)$ konnten so kulturell Keime nachgewiesen werden. Bei 238 Pat. $(=70,8 \%)$ lag eine Monoinfektion vor, bei 65 Pat. $(=19,4 \%)$ eine Mischinfektion mit Nachweis von insgesamt 144 Keimen. Dabei wurde folgende Erreger-Verteilung - geordnet nach Mono-/Misch-Infektion - gefunden: grampositive Keime $32,5 \% / 17,3 \%$; gramnegative Keime 19,9\%/8,9\%; anaerobe Keime 6,8\%/9,1\%; Mycobacterium tuberculosis 3,1\%/2,4\%.

Bei 139 Pat. $(=52,2 \%)$ war - oft ohne korrekte Diagnose bereits extern eine Vorbehandlung erfolgt: 104mal mit Antibiotika, 21 mal durch ein- oder mehrmalige Pleurapunktionen, 14mal mittels einer Pleuradrainage. Die Dauer der auswärtigen Vorbehandlung bezogen auf die klare Manifestation des Empyems war in der Regel den Verlegungsberichten nicht eindeutig zu entnehmen, so daß eine statistische Auswertung unterbleiben muß. Diesbezüglich ist auch der Zeitpunkt der Diagnosestellung nicht hilfreich, da erfahrungsgemäß eine breite Latenzzeit im prästationären, aber auch stationären Krankheitsverlauf angenommen werden. Wegen häufig unklarer initialer Symptomatik sind auch die anamnestischen Angaben der Pat. zur definitiven Feststellung des Erkrankungsbeginns nicht sicher verwertbar.

In Anlehnung an die gängigen Angaben in der Literatur wurden die folgenden, komplizierenden Befunde differenziert und in der in Klammern angegebenen Häufigkeit (bezogen auf 336 Pat.) festgestellt: Bronchopleurale Fistel $(n=81$ [24,1\%]), multipel gekammertes Pleuraempyem $(n=68$ $[20,2 \%])$, „gefesselte Lunge“ ( $n=28$ [8,3\%]), kalzifizierte Pleurahöhle $(\mathrm{n}=18[5,4 \%])$, Bronchusstumpf-Insuffizienz nach Pneumonektomie $(\mathrm{n}=25[7,4 \%])$, ösophagopleurale Fistel $(\mathrm{n}=2[0,6 \%])$.

In 316 Fällen (94\%) konnten die Pat. geheilt aus der stationären Behandlung entlassen werden. Nur 5 Pat. wurden überwiegend wegen fortgeschrittenem Tumorleiden - ungeheilt mit Dauerdrainage entlassen und 15 Pat. $(=4,5 \%)$ verstarben im Verlauf der stationären Behandlung vorwiegend an den Folgen der primären Erkrankung (z.B. Malig- 
nom). Bezogen auf die Genese der Empyeme sind in Tab. 1 die durchgeführten Maßnahmen benannt. Dabei konnte bei 257 Pat. (=76,5\%) durch eine Drainagebehandlung mit Spülung und Fibrinolyse die Ausheilung erreicht werden.

\section{Methodik (Spül-Drainage / Fibrinolyse)}

Im genannten Zeitraum wurde die Spülbehandlung mehrfach modifiziert und danach in der jeweils neuen Technik bei allen konsekutiv behandelten Pat. angewendet: Bei insgesamt 38 Pat. in den Jahren 1986-1988 wurde eine einfache Pleuradrainage plaziert; bei den ersten 14 Fällen ohne Fibrinolyse, bei den folgenden 24 Pat. mit intrathorakaler Instillation von Streptokinase/Streptodornase zur Fibrinolyse (Varidase $\mathrm{N}^{\circledR}$, [Fa. Lederle, Münster]). Seit 1989 wurde die „doppelläufige“ Spüldrainage verwendet, zunächst durch Plazieren von 2 Drains möglichst im apikalen Höhlenpol (Einspül-Kanal) bzw. basal (Ablauf-Kanal). Seit 1990 werden routinemäßig handelsübliche, mehrfach perforierte Doppellumen-Drains verwendet mit einem an der Spitze mündenden Spülkanal (Doppellumiger Trokarkatheter [Fa. Argyle/Sherwood Medical $\mathrm{GmbH}$, Steinbach]). Nur bei 5 Pat. erfolgte keine Fibrinolyse; die übrigen „doppellumig“ drainierten Pat. wurden fibrinolytisch behandelt.

Nach Sicherung der klinischen und röntgenologischen Verdachtsdiagnose durch eine Probepunktion wird die günstigste Stelle für das Einlegen eines bzw. zweier Pleuradrains vorzugsweise unter Röntgendurchleuchtung markiert. Seit Verfügbarkeit der Computertomographie (CT) wird die Lokalisation in den meisten Fällen erfolgreich anhand aktueller CTScans vorgenommen; aus räumlich-technischen Gründen wurde jedoch in keinem Fall CT-gesteuert punktiert oder drainiert. In einzelnen Fällen - erfahrungsgemäß ist dies wegen häufig hoher Dichte des Sekrets oft unbefriedigend konnte die günstigste Punktions-/Drainage-Stelle sonographisch bestimmt werden. Mittels Röntgenkontrastmittel kann die Ausdehnung der Empyemhöhle bestimmt, sowie - nötigenfalls durch Wechsel von Lagerung und Position des Pat. eine bestehende Kammerung bzw. eine bronchopleurale Fistel festgestellt werden. Die Einlage der Drainage(n) erfolgt unter sterilen Kautelen in Lokalanästhesie. In Kontroll-Röntgenaufnahmen wird grundsätzlich die korrekte Lage des/der Drains kontrolliert und dokumentiert. Es wird ein Wasserschloß- (Bülau-Prinzip) oder ein Dauersog-Drainagesystem (normalerweise $-20 \mathrm{~cm} \mathrm{H}_{2} \mathrm{O}$ ) zur kontinuierlichen, geschlossenen Drainage des Pleurasekretes bzw. des Eiters und zur Ableitung der Spülflüssigkeit angeschlossen; dies kann evt. bei Verminderung der Eiterproduktion zur besseren Mobilisierung der Pat. stundenweise entfernt werden. Bei unabhängigen Kammern kann initial oder im Verlauf die Einlage zusätzlicher Drains bzw. bei Schwierigkeiten bei der Spülung ggf. ein mehrfacher Drainwechsel nötig werden.

Regelmäßig wird nach initialer Evakuierung des Pleuraraumes eine Spülung mit 500-1000 ml steriler Kochsalz-Lösung - bis zur Klärung der Spülflüssigkeit - vorgenommen. Im weiteren wird täglich einmal, initial bei Bedarf auch zweimal, mit jeweils 500-1000 ml physiologischer Kochsalz-Lösung evtl. unter Zusatz eines lokalen Desinfektionsmittels (z.B. Betaisodona-Lösung ${ }^{\circledR}$ [Fa. Mundipharma, Limburg/Lahn]) gespült. Danach werden täglich 1 bzw. 2 Fl. Varidase ${ }^{\circledR}$ Trokkensubstanz (= Streptokinase 100000 IE \& Streptodornase
$25000-100000$ IE pro Fl.) in jeweils $20-30 \mathrm{ml}$ isotonischer Kochsalz-Lösung gelöst, in die Höhle installiert und die Drains für 3-6 Std. abgeklemmt. Dies wird fortgesetzt, solange purulentes oder flockiges Sekret gefördert wird und/oder bei einer röntgenologisch feststellbaren Befundbesserung mit $\mathrm{Zu}-$ nahme der Lungenausdehnung noch Auflagerungen auf der „gefesselten“ Lunge vorhanden sind. Zusätzlich erfolgt normalerweise eine resistenzgerechte Antibiotika-Therapie für ca. 7 Tage.

Der Behandlungsfortschritt wird durch regelmäßige konventionelle Thorax-Röntgenaufnahmen kontrolliert und dokumentiert. Klinisch zeigt sich normalerweise innerhalb weniger Tage eine augenfällige Besserung des Allgemeinzustands und der Infektions-Symptomatik mit Normalisierung der Körpertemperatur und der infektionstypischen Laborwerte sowie eine Rückbildung der Verschattung im Röntgenbild. Nach Sistieren der eitrigen Sekretion und Rückgang der Sekretmenge auf weniger als $50 \mathrm{ml}$ wird nach Absetzen der systemischen oder lokalen Antibiose das spontan abfließende oder mit steriler NaCl-Lösung ausgespülte Pleurasekret an drei verschiedenen Tagen bakteriologisch untersucht und die Drains nach 3malig negativer Kultur entfernt. In der Folgezeit wird die Befundentwicklung stationär - bei problemloser Nachsorge auch ambulant - klinisch und röntgenologisch kontrolliert, um ein normalerweise nach etwa 10 Tagen erkennbares Empyemrezidiv auszuschließen.

Bei röntgenologisch relevanter Pleuraschwiele nach Abschluß der Drainagetherapie wird mittels Bodyplethysmographie und Perfusions-Szintigraphie vor Entlassung aus der stationären Behandlung das Ausmaß einer eventuellen Funktionsstörung bestimmt. Eine Indikation zur operativen Sanierung (Dekortikation) sehen wir nur bei persistierenden, eindeutig pathologischen Werten (Vitalkapazität $<75 \%$ des Sollwertes) bei einer Routine-Befundkontrolle nach 4-6 Monaten, da die Möglichkeit einer spontanen Normalisierung durch intensive Atemgymnastik genutzt werden soll. In den meisten Fällen ist tatsächlich zu diesem Zeitpunkt eine weitgehende „Restitutio ad integrum“ zu beobachten, so daß damit keine Indikation für ein operatives Vorgehen mehr besteht.

Ein Versagen der beschriebenen Spüldrainage-Behandlung konstatieren wir bei inkompletter Lungenausdehnung mit Resthöhlenbildung und persistierendem Nachweis pathogener Keime im Pleurasekret ohne weitere Besserungstendenzen nach der zweiten Behandlungswoche bzw. beim Verbleib einer massiven Pleuraschwiele mit Schrumpfungszeichen. Dies erfordert üblicherweise die Durchführung einer Empyemektomie mit Dekortikation bzw. bei zustandsbedingt inakzeptablem Operationsrisiko einer palliativen Thoraxfensterung, der wir gegenüber einer Dauerdrainage den Vorzug geben, da sie die Patienten auf Dauer deutlich geringer beeinträchtigt.

\section{Ergebnisse (metapneumonisches Pleuraempyem)}

Beim metapneumonischen Pleuraempyem kann in weitaus den meisten Fällen (92,2\%) durch eine Drainagebehandlung mit Spülung eine endgültige und rezidivfreie Ausheilung erreicht werden (siehe Abb.5). Durch Modifikation in der Anwendung zweier Drainagen bzw. zusätzlichem Einsatz von Fibrinolytika ist vor allem eine deutliche Verkürzung der 


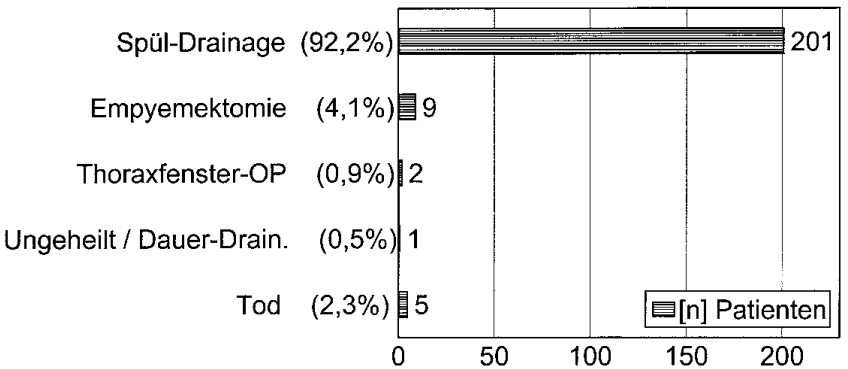

Abb. 5 Metapneumonische Pleuraempyeme $(n=218)$. Behandlungsergebnisse.

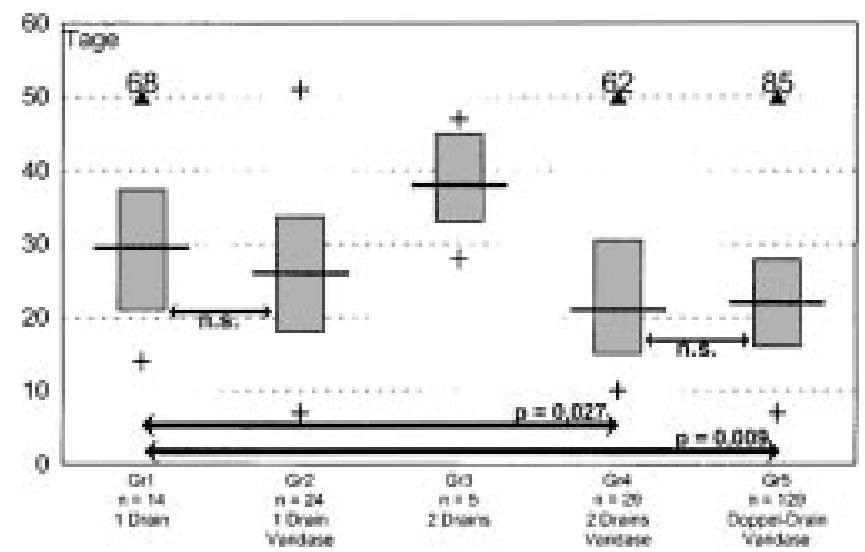

Abb. 6 Metapneumonische Pleuraempyeme $(n=218)$. Behandlungsdauer bei verschiedenen Drainage-Verfahren $(n=201)$. Darstellung in Boxplot-Graphik; Median; 25\%-/75\%-Quartil, Min.-/Max.-Wert sowie p-Wert im Gruppenvergleich (n. s. = nicht signifikant).

Drainagezeiten zu erreichen. Bei einer in dieser Gruppe beobachteten Mortalität von 2,3\% und der Versorgung eines einzigen Patienten mit Dauerdrainage wurde nur bei 11 Pat. (5\%) eine operative Sanierung nötig: Bei 9 Pat. wurde dabei eine Empyemektomie und Dekortikation, zweimal eine Fensterungsoperation bei polymorbiden Pat. durchgeführt. Fibrinolytika wurden jeweils einmal täglich durchschnittlich an 12,96 (6 - 30) Tagen instilliert.

Für die erfolgreich mittels Drainage therapierten Pat. mit metapneumonischem Pleuraempyem $(n=201)$ wurden bei retrospektiver Auswertung - im Sinne einer historischen Fallkontrollstudie - Pat. mit unterschiedlicher Behandlungsmodalität zusammengefaßt und hinsichtlich der Therapiedauer (= Drainage-Zeit) statistisch verglichen. Dabei wurden - entsprechend der chronologischen Weiterentwicklung des Verfahrens (siehe „Methodik“) - folgende Gruppen (Gr) zusammengefaßt: Gr1 - eine Drainage/keine Fibrinolyse $(n=14)$; Gr2 - eine Drainage/mit Fibrinolyse $(n=24)$; Gr3 zwei Drainagen/keine Fibrinolyse $(n=5)$; Gr4 - zwei Drainagen/mit Fibrinolyse $(n=29)$; Gr5 Doppellumen-Drainage/mit Fibrinolyse $(\mathrm{n}=129)$.

Im Vergleich zeigt sich eine - nicht signifikante - Verkürzung der durchschnittlichen Therapiedauer bei Pat. mit EinfachDrainage durch Fibrinolyse von 31,8 in der Gr1 auf 26,5 Tage in der Gr2 $(p=0,231)$, die sich durch Anwendung eines
Doppellumen-Prinzips und Kombination mit Fibrinolyse in den Gruppen Gr4 bzw. Gr5 auf Mittelwerte von 24,0 bzw. 23,7 Tage weiter reduzieren läßt. In Abb. 6 sind als BoxplotGraphik die jeweiligen Medianwerte, die 25\%/75\%-Quartile und die Minimal-/Maximal-Werte dargestellt. Signifikante Unterschiede zwischen den Gruppen sind im hier geeigneten U-Test nach Mann-Whitney festgestellt und mit den jeweiligen p-Werten dargestellt. Es zeigt sich mit einem p-Wert von 0,027 ein schwach signifikanter Unterschied zwischen Gr1 und $\mathrm{Gr} 4$ und ein signifikanter Unterschied zwischen Gr1 und Gr5 ( $p=0,009$ ). Obwohl die Gr3 (Zwei Drainagen/keine Fibrinolyse) mit 38,8 Tagen den weitaus höchsten Mittelwert aufweist, ergibt sich - wohl begründet durch die kleine Patientenzahl $(\mathrm{n}=5)$ - statistisch keine Signifikanz.

Auffällige lange Behandlungszeiten sind erfahrungsgemäß bei auswärts invasiv vorbehandelten Patienten nötig (z.B. nach mehrfachen „therapeutischen“ Punktionen oder längerer Drainagetherapie). Werden diese Pat. $(n=30)$ in einer gesonderten Gruppe (GrV) zusammengefaßt und die jeweiligen Gruppen um diese Pat. bereinigt (Gr1b: - 1 Pat.; Gr4b: -4 Pat.; Gr5b: - 25 Pat.) ergibt sich für die GrV tatsächlich eine vergleichsweise lange mittlere Behandlungszeit von 40,6 Tagen. Korrespondierend verkürzt sich die Dauer für die bereinigten Gruppen Gr4b und Gr5b um etwa 4 Tage auf 20,6 bzw. 19,8 Tage. Die so resultierenden Behandlungszeiten sind in Abb. 7 wiederum mit Boxplot-Graphik (Median; 25\%-/75\%Quartile; Minimum/Maximum) dargestellt. Mit einem jeweiligen p-Wert <0,001 ist die Behandlungsdauer zwischen Gr4b und GrV und zwischen Gr5 und GrV hochsignifikant unterschiedlich. Durch den Ausschluß vorbehandelter Pat. und entsprechender Verkürzung der Behandlungszeiten in den Gruppen Gr4b und Gr5b werden die Unterschiede zu den beiden $1 \mathrm{fach}$ drainierten Gruppen mit mindestens einem Trend (Gr2 vs. Gr4b: p=0,085) statistisch auffällig (siehe Abb. 7).

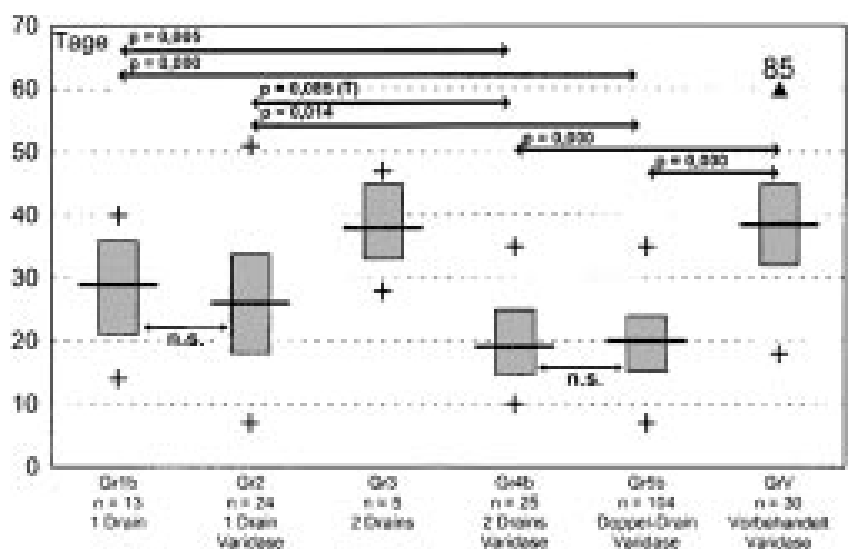

Abb. 7 Metapneumonische Pleuraempyeme $(n=218)$. Differenzierte Behandlungsdauer bei verschiedenen Drainage-Verfahren $(n=201)$ bei gesonderter Berücksichtigung auswärts invasiv vorbehandelter Patienten. Darstellung in Boxplot-Graphik: Median, 25\%-/75\%-Quartil, Min.-/Max.-Wert sowie p-Wert im Gruppenvergleich (n.s. = nicht signifikant). 


\section{Diskussion}

Bei Gruppierung aller behandelten Empyemfälle nach ihrer Genese erscheint die eingehendere Betrachtung des zahlenmäßig häufigsten „Metapneumonischen Empyems“ sinnvoll, da es - wenn auch nicht häufig - im normalen Krankengut eines Allgemein-Krankenhauses auftreten kann, während z.B. postoperative Empyeme oder Pneumolysen- bzw. Spät-Empyeme kaum je außerhalb spezialisierter Fachabteilungen gesehen und behandelt werden. Zudem können durch Konzentration auf ein relativ homogenes Krankheitsbild die Behandlungsergebnisse mit Berichten in der Literatur verglichen werden. Mit 218 behandelten Pat. im Beobachtungszeitraum von 10 Jahren und weitgehend standardisierten Behandlungsverfahren ist eine fundierte Analyse möglich, zumal die Modifikation in der technischen Durchführung chronologisch erfolgte und bei identischer Behandlung aller Pat. in definierbaren Zeiträumen interne Vergleichbarkeit besteht. Um Einwänden vorzubeugen erscheint die Feststellung nötig, daß keinerlei Selektion vorgenommen wurde und alle zugewiesenen Empyem-Patienten stadienunabhängig gleichartig behandelt und in der vorliegenden Studie berücksichtigt worden sind.

Mit einer Heilungsrate beim „Metapneumonischen Empyem“ von $97,2 \%$ - davon in 92,2\% der Fälle mittels Drainagetherapie - und einer Mortalität von 2,3\% sind unsere Ergebnisse, verglichen mit Berichten in neuesten Publikationen, beachtenswert. Häufig findet sich bei Diskussion der Ergebnisse in der Literatur der Hinweis auf eine hohe Rate komplizierender Risiken, die jedoch auch in unserem Krankengut in ähnlicher Häufigkeit festzustellen sind: In $21 \%$ der Fälle waren die Pat. über 70 Jahre, in fast $10 \%$ über 80 Jahre alt (Abb.1); bei allen mit Drainage ausgeheilten Pat. $(n=257)$ lagen in $30,4 \%$ der Fälle Lungenparenchymfisteln, in $23 \%$ multiple Kammerungen und in 7,4\% eine "gefesselte“ Lunge vor und im Patientengut fanden sich $51 \%$ Raucher, $29,3 \%$ der Pat. tranken „viel“ Alkohol bzw. waren alkoholkrank, bei $15,4 \%$ der Pat. bestand eine maligne Grunderkrankung, bei 6\% ein Diabetes und 5,3\% der Pat. standen unter einer Steroid-Dauertherapie.

Die Betrachtung der Therapiedauer erlaubt eine gut fundierte Aussage zur „optimalen“ Drainagebehandlung, nachdem statistisch auffällige Unterschiede zwischen den verschiedenen Behandlungsmodalitäten gefunden werden: Eine intrapleurale Fibrinolyse mit Varidase ${ }^{\circledR}$ ist verbunden mit einer Verkürzung der Therapiedauer. Bei Kombination der Fibrinolyse mit einer „doppelläufigen“ Drainage ergibt sich eine signifikante Verkürzung, die besonders augenfällig wird bei Abgrenzung invasiv vorbehandelter Patienten. Dabei zeigen fast identische Behandlungszeit und hoher p-Wert im statistischen Vergleich ( $p=0,896)$, daß eine zweilumige Einzeldrainage (Gr5) verglichen mit 2 plazierten Drains ( $\mathrm{Gr} 4)$ gleichwertig und bei einfacherer Technik für die Pat. vorteilhafter ist. Dies, wie auch die signifikante Verkürzung der Behandlungsdauer, unterstützt die früher formulierte These, daß bei „richtiger Anwendung ... trotz Spülung innerhalb der Empyemhöhle kein Überdruck, sondern ... ein deutlicher Unterdruck herrscht ... . Hierdurch wird die Lungenausdehnung gefördert. Gleichzeitig wird vermieden, die Höhle täglich neu aufzudehnen und so eventuelle Verklebungen oder Verwachsungen, die zur Höhlenschrumpfung notwendig sind, zu lösen, wie es bei der Einwegspülung von Hand sicher oft geschehen dürfte.“ [10].
Schon damals wurde eine Instillation von Fibrinolytika durchgeführt. Der günstige Effekt dieser Maßnahme wird auch jetzt erkennbar: Wenngleich nur in 5 Fällen eine Behandlung mit 2 Drains ohne Fibrinolyse durchgeführt wurde, zeigen die deutlich längeren Behandlungszeiten in dieser Gruppe auch ohne statistischen Signifikanznachweis eindrucksvoll den Wert der zusätzlichen Fibrinolyse.

Bei gesonderter Betrachtung auswärts invasiv vorbehandelter Pat. ergeben sich statistisch signifikant längere Behandlungszeiten, was vor dem Hintergrund des regelhaften, zeitabhängigen Krankheitsverlaufs - mit Fortschreiten von der exsudativen über die fibrinopurulente bis in die Organisations-Phase - nicht verwundert. Dem entspricht die einhellige Aussage in der Literatur, daß der therapeutische Aufwand hin zu eingreifenderen Verfahren mit zunehmender Dauer der Erkrankung steigt. Dies rechtfertigt sicher die nachdrückliche Forderung nach verzögerungsfreier Einleitung einer kompetenten und stadiengerechten Therapie in einer geeigneten Institution, die darüber hinaus in der Lage ist, Komplikationen zu behandeln bzw. bei Therapieversagen auf operative Maßnahmen „umzusteigen“. Für alle optionalen Behandlungsverfahren gilt jedoch, daß sie sich am „Erreichbaren“ - bezogen auf Heilungsquote, Morbidität und Mortalität - messen lassen müssen.

Ein Vergleich unserer Ergebnisse mit Angaben in der Literatur ist nur bedingt möglich, da sich die Behandlungsstrategien kaum vergleichen lassen. Immerhin wird in der Literatur des letzten Jahrzehnts die konservative Drainagetherapie nach langjähriger Dominanz operativer Verfahren wieder neu gewürdigt. Es entspricht unserer bisherigen Auffassung, wenn an einer Stelle ausdrücklich festgestellt wird, es sei für den Patienten aus „Sicht der Morbidität und der Kosten ... vorteilhaft, ... mit geschlossener Drainage behandelt zu werden ... anstelle invasiverer chirurgischer Verfahren wie Thorakoskopie, Standard-Thorakotomie oder offene Drainage“ [28].

Beschrieben werden Vorteile einer mit bildgebenden Verfahren überwachten Plazierung von Drains; ohne nähere Angaben $\mathrm{zu}$ weiteren Behandlungsmodalitäten werden in einer Übersichtsarbeit Heilungsraten von $72 \%$ bis $92 \%$ (Durchschnitt: $81 \%$ ) gegenüber einer mittleren Erfolgsrate von $47 \%$ bei konventionell drainierten Pat. genannt [25]. Für die konventionelle Drainagetherapie wird jedoch schon 1979 in einer Arbeit aus unserer Klinik eine Heilung in $70 \%$ der Fälle beschrieben, mit allerdings relativ langen Drainagezeiten (60\% > 21 Tage, $44 \%>28$ Tage, $8 \%>56$ Tage) und einer hohen Letalität (15\%) [11]. Die Kombination der bildgesteuerten Drain-Einlage mit intrapleuraler Anwendung von Fibrinolytika schließlich war in einer anderen Studie in 94\% der Fälle ( $n=118$ ) erfolgreich; einschränkend ist festzustellen, daß hier nur 79mal ein Empyem, bei einem Drittel der Pat. jedoch sterile Pleuraergüsse bzw. Hämatothoraces behandelt wurden [22]. Ähnliche Ergebnisse (92\% Heilung) werden beschrieben bei randomisierter Anwendung von Streptokinase versus Urokinase bei Empyemen $(n=11)$ und parapneumonischen, gekammerten Pleuraergüssen $(n=39)$ [5]. In einer randomisierten und kontrollierten Studie werden für die Anwendung von Streptokinase zur Fibrinolyse gegenüber einer Spülung mit NaCl-Lösung die Ausheilung aller Fibrinolyse-Fälle ohne chirurgische Maßnahmen (100\% Heilung vs. $75 \%$ in der Kontrollgruppe, $n=24$ ) und weitere, statistisch signifikante Vor- 
teile beschrieben [7]. In anderen, kleineren Serien wurden Erfolge der Fibrinolyse-Behandlung in einer Häufigkeit von $62 \%$ [37], 72\% [16], 77\% [27], 79\% [3], 85\% [4] und 92\% [14] erreicht.

Der Einsatz eines doppellumigen Drainageverfahrens ist bisher nur selten publiziert $[15,25]$. Die günstigen Ergebnisse unseres Behandlungsverfahrens mit kombinierter Anwendung von Fibrinolytika und Doppellumen-Drainage beim manifesten Pleuraempyem - immerhin in ca. 30\% der Fälle bei gekammertem Empyem oder gar „gefesselter Lunge“ legen bei Berücksichtigung der statistischen Auffälligkeiten und der zitierten Literatur den Schluß nahe, daß ein synergistischer Effekt die hohe Erfolgsrate ermöglicht. Es kann vermutet werden, daß die publizierten geringen Erfolgsraten der „geschlossenen“ Pleuradrainage bei optimierter - und wohl auch geduldigerer - Drainagetherapie hätten gesteigert werden können; diese Auffassung wird auch in der Literatur vertreten [28]. Sicher jedoch wird die strikte BehandlungsPrämisse widerlegt, daß „kein gekammertes Empyem durch Drainage geheilt werden kann“ und in diesen Fällen eine laterale Thorakotomie bzw. alternativ eine video-assistierte thoraxchirurgische Operation (VATS) indiziert sei [20].

Zwar verweisen die Befürworter der frühen, „offenen“ Behandlung des Pleuraempyems auf günstige Resultate und verschiedentlich auch auf relativ kurze stationäre Behandlungszeiten; über die - nach eigener Erfahrung - durchaus relevante postoperative Morbidität, die Komplikationsraten, die Operationsrisiken (u.a. Transfusionsrisiken) und die oft jahrelang anhaltenden Beschwerden wird jedoch selten berichtet. In den meisten Arbeiten fehlt die Angabe, ob Pat. als „inoperabel“ von operativen Maßnahmen ausgeschlossen wurden und wie das Vorgehen in diesen Fällen war. Doch allein die - leider nicht immer klar benannte oder zuzuordnende - publizierte perioperative Mortalität spricht für die Vermeidung chirurgischer Maßnahmen, sofern eine konservative Ausheilung möglich ist. Genannt oder ermittelt finden sich in der Literatur postoperative Mortalitätsraten von $6,2 \%$ bei Dekortikation und $21 \%$ bei „Offener Drainage“ [1], eine 30-Tage-Letalität von 7,4\% [2], bzw. Hospital-Mortalitäten von $8 \%$ [24], 15\% [23], 16\% [31], 19,8\% [29] bis 33\% [26]. Selbst für die VATS werden Mortalitätsraten von 4\% [36], 6,6 [17], $9 \%$ [40] und $13 \%$ [26] beschrieben.

Die Dauer der Drainagebehandlung liegt mit maximal 68 Tagen (Gr1b), 51 Tagen (Gr2), 47 Tagen (Gr3), jeweils 35 Tagen (Gr4b/Gr5b) und 85 Tagen (GrV) in unserem Kollektiv vergleichsweise hoch. Dies kann einerseits begründet werden mit der Zusammensetzung des Patientengutes mit einzelnen, inoperablen Patienten ( $\mathrm{z}$ B. polymorbide Pat. in einem Lebensalter von $>90$ Jahren $[n=8]$ bzw. 23 Pat. mit maligner Pleura-Erkrankung) bzw. mit der Verweigerung operativer Maßnahmen durch die Pat. oder Angehörige. Andererseits kann sicher auch ein - gemessen an publizierten Verfahren möglicherweise zu restriktives und übervorsichtiges Vorgehen beim Abschluß der Drainagebehandlung als Grund angeführt werden: Die oft beschriebene, sehr frühe und meist komplikationslose Entfernung der Drainagen ohne verzögernde „Rituale“ nach Thorakotomie/VATS gibt Anlaß, das bisherige Verfahren zu überdenken. Es finden sich jedoch auch in der Literatur vergleichbar lange maximale Behandlungsdauern nach operativen Maßnahmen (z.B. 84 Tage [20] bzw. 140
Tage [6]). Immerhin dürfte bei Berücksichtigung auch protrahierter Verläufe mit Komplikationen, Rezidiven oder Mehrfacheingriffen die durchschnittliche stationäre Verweildauer bei Thorakotomie mit Vor- und Nachbehandlung wohl nicht relevant kürzer sein als die von uns zuletzt (Gr5b) beobachteten Behandlungszeiten. Diesbezüglich ist zu beachten, daß in Publikationen häufig nur die postoperative Drainagezeit und/oder stationäre Behandlungsdauer benannt wird. Bei vergleichender Betrachtung ist jedoch die Gesamtdauer der stationären Behandlung zu berücksichtigen; sie wird in zwei Arbeiten mit durchschnittlich 33,7 (13-84) Tagen für die Thorakotomie [20] und für die VATS mit 16,3 (8-36) Tagen [30] bzw. 18,3 (9-32) Tagen [20] angegeben.

Seit Einführung der minimal-invasiven Operationsmethoden in der Thoraxchirurgie wurde bei Beschreibung und Bewertung der neuen Methoden auch über den möglichen Einsatz der video-assistierten Thoraxoperation (VATS) beim Pleuraempyem berichtet $[9,12,18,35]$. Dabei tritt diese Methode an die Stelle der schon früher als effektiv und risikoarm propagierten Thorakoskopie [21,26,32]. Anläßlich einer Ende 1995 durchgeführten Umfrage bei 229 Thoraxchirurgen zum Stellenwert der VATS bei verschiedenen thorakalen Erkrankungen wurde bei insgesamt 190 Antworten mehrheitlich festgestellt, die Methode sei bei der Behandlung des „frühen Empyems“ „akzeptabel“ ( $\mathrm{n}=112$ ); 52mal wird sie als „bevorzugtes“, 16mal als „experimentelles“ und 10mal als „inakzeptables" Verfahren deklariert [19]. Inzwischen sind weitere Berichte mit zunehmenden Fallzahlen publiziert und wohl abhängig von der Größe und Zusammensetzung des Patientengutes - für die VATS beim Empyem im fibrinopurulenten Stadium unterschiedliche Erfolgsraten beschrieben: $72 \%$ ( $n=67$ von 88 Pat. nach Selektion und primärer Dekortikation in 21 Fällen [36], $82 \%(n=45$ von 52 Pat. nach Selektion und primärer Dekortikation in 7 Fällen) [6] und $100 \%(n=10)$ [30]. Dabei wird in diesen Publikationen für alle Pat. eine klare Indikation für eine primär-operative Behandlung festgestellt und die VATS statt einer „notwendigen“ Dekortikation durchgeführt. Wenig fundiert und möglicherweise irreführend erscheinen hierbei die Feststellungen, alle Pat. seien „initial mit Drainage und Antibiotika“ behandelt worden [36] bzw. eine Behandlung mit „Drainage, Fibrinolyse und Antibiotika“ sei gescheitert [6], nachdem dies - hinsichtlich der Technik und der Dauer nicht näher differenziert - „auswärts“ und somit nicht in der weiterbehandelnden Fachklinik durchgeführt wurde. Selbst nach initialer Selektion und Ausschluß von 21 (=24\%) bzw. 7 Pat. (=13\%) mit primär durchgeführter Thorakotomie und Dekortikation wurde somit bei VATS nicht selten ein Umsteigen zur konventionellen Thorakotomie nötig (Konversionsrate: 10\% [20], 18\% [6], 28\% [36] und auch in anderen Serien bleibt die primäre Erfolgsrate mit 60\% [26] bzw. 83\% [17] bei zudem relevanter Letalität (13\% bzw. 6,6\%) deutlich hinter unseren Ergebnissen (Heilung: 92,2\%, Letalität $2,3 \%$ ) zurück. Wenngleich ein VATS-Eingriff zunächst als „wenig belastend“ deklariert wird, wird neben den immanenten Risiken folgerichtig immer auch - nach unserer Meinung oft vermeidbar - das Risiko und die Auswirkung einer Thorakotomie und Dekortikation in Kauf genommen.

In bisher einer einzigen Veröffentlichung wird über eine randomisierte und kontrollierte Untersuchung berichtet, die beachtliche Vorteile einer VATS-Behandlung im Vergleich zu einer Drainage-/Fibrinolyse-Behandlung zeigt [40]. An kleinen 
Patientengruppen (VATS: $n=11$, Fibrinolyse: $n=9$ ) wird ein „primärer Erfolg“ in 100\% bzw. $44 \%$ der Fälle beschrieben. Dies wird allerdings deutlich relativiert durch ein rigides Studiendesign zuungunsten der Fibrinolysegruppe, bei der nach nur 3tägiger Behandlung ein Behandlungs-Mißerfolg festgestellt wurde, wenn das Thoraxröntgenbild nicht eine Abnahme um $>50 \%$ des initialen Erguß-Volumens zeigte, bzw. die Körpertemperatur nicht unter $38^{\circ} \mathrm{C}$ und die Leukozytenzahl nicht unter $11000 / \mu l$ abgefallen war. In diesen Fällen (5 von 9 Pat.) wurde unmittelbar eine VATS durchgeführt und bei Ermittlung der - somit für die Fibrinolyse etwas höheren - Behandlungskosten wurden die dabei angefallenen Folgekosten addiert. Explizit wird dies auch in einer Review-Arbeit bemängelt und festgestellt, es scheine, daß ,.... the “deck was stacked" against the streptokinase-group ..." [28]. Nach unseren Erfahrungen sind die verwendeten Kriterien zudem ungeeignet: Die Abnahme einer pleuralen Verschattung im Röntgenbild ist aus technischen Gründen kaum zu messen, eine Fibrinolyse ist - bekanntermaßen - häufig verbunden mit einem Anstieg der Körpertemperatur (in unserem Krankengut zeigten $44 \%$ der Pat. einen Anstieg um mindestens $1^{\circ} \mathrm{C}$ bis maximal $3^{\circ} \mathrm{C}$ nach Instillation) und die absolute Leukozytenzahl ist bereits bei Behandlungsbeginn recht unterschiedlich (siehe Abb. 4).

Wir glauben, das vorgestellte Verfahren einer DoppellumenDrainage mit Spülung und Einsatz proteolytischer Enzyme zur Fibrinolyse aufgrund unserer guten Erfahrungen für die Behandlung der meisten Formen des Pleuraempyems, grundsätzlich jedoch für das metapneumonische Pleuraempyem empfehlen zu können. Dies sollte unmittelbar in geeigneten und erfahrenen Institutionen eingeleitet und durchgeführt werden, da dort die möglichen Komplikationen adäquat erkannt und behandelt, und nötigenfalls bei unbefriedigendem Ergebnis oder Therapieversagen stufenweise eskalierend alle optionalen operativen Maßnahmen mit vertretbarem Risiko für die betroffenen Pat. durchgeführt werden können.

Es ist nicht auszuschließen, daß eine primäre Behandlung per VATS als konkurrierend operatives Verfahren günstigere Ergebnisse zeigt; bisher ist dies keinesfalls zweifelsfrei nachgewiesen. Unsere Ergebnisse ermutigen zu einem tendenzfreien Vergleich beider Verfahren in einer randomisierten Studie an einem vergleichbaren, unselektierten Krankengut mit optimaler und unlimitierter Anwendung beider Verfahren. Bei definierten, gleichartigen Bedingungen für den Abschluß einer Behandlung und klaren Erfolgskriterien (z.B.: Drainagedauer, stationäre Behandlungsdauer, Morbidität, Mortalität, primäre Heilung, Rezidivfreiheit innerhalb eines geeigneten Zeitraumes [z.B. 3 Monate], additive Verfahren und deren Auswirkungen, direkte und indirekte Behandlungskosten sowie Folgekosten innerhalb eines Zeitraumes von ca. 1 Jahr) sollte sich an einem ausreichend großen Patientengut der vorteilhaftere Weg erkennen lassen.

\section{Literatur}

${ }^{1}$ Ashbaugh DG. Empyema Thoracis - Factors Influencing Morbidity and Mortality. Chest 1991; 99: $1162-1165$

${ }^{2}$ Bauer H.-G, Probst G, Bauer E, Vogt-Moykopf I. Results and Complications in the Management of Acute and Chronic Pleural Empyema - Retrospective Analysis of the Past Five Years.
Langenbecks Arch Chir 1989; (Suppl. 2) : 211 - 217 (Kongreßbericht)

${ }^{3}$ Bergh NP, Ekroth R, Larsson S, Nagy P. Intrapleural Streptokinase in the Treatment of Haemothorax and Empyema. Scand J Thor Cardiovasc Surg 1977; 11: 265 - 268

${ }^{4}$ Bouros D, Schiza S, Panagou P, Drositis J, Siafakas N. Role of Streptokinase in the Treatment of Acute Loculated Parapneumonic Pleural Effusions and Empyema. Thorax 1994; 49: 852 855

${ }^{5}$ Bouros D, Schiza S, Patsourakis G, Chalkiadakis G, Panagou P, Siafakas NM. Intrapleural Streptokinase versus Urokinase in the Treatment of Complicated Parapneumonic Effusions: A Prospective, Double-blind Study. Am J Respir Crit Care Med 1997; 155 : $291-295$

${ }^{6}$ Cassina PC, Hauser M, Hillejan L, Greschuchna D, Stamatis G. Video-assisted Thoracoscopy in the Treatment of Pleural Empyema: Stage-based Management and Outcome. J Thorac Cardiovasc Surg 1999; 117: $234-248$

${ }^{7}$ Davies RJO, Traill ZC, Gleeson FV. Randomised Controlled Trial of Intrapleural Streptokinase in Community Acquired Pleural Infection. Thorax 1997; 52: 416 - 421

${ }^{8}$ Ferguson AD, Prescott RJ, Selkon JB, Watson D, Swinburn CR. The Clinical Course and Management of Thoracic Empyema. QJM 1996; 89: 285 - 289

${ }^{9}$ Ferguson MK. Thoracoscopy for Empyema, Bronchopleural Fistula and Chylothorax. Ann Thorac Surg 1993; 56: 644 - 645

${ }^{10}$ Frey DJM, Stuber U, Wolfart W. Doppelläufige Spüldrainage zur Behandlung des Pleuraempyems. Fortbildung in Thoraxkrankheiten. Stuttgart: Hippokrates Verlag, 1980. Bd. 9:99 - 110

${ }^{11}$ Gabler A, Liebig S, Heidbüchel H.-P, Warlies F. Ergebnisse der Dauersaugdrainagebehandlung beim Pleuraempyem. Prax Pneumol 1979; 33: 536 - 540

${ }^{12}$ Hurley JP, McCarthy J, Wood AE. Retrospective Analysis of the Utility of Video-Assisted Thoracic Surgery in 100 Consecutive Procedures. Eur J Cardiothorac Surg 1994; 8: 589 - 592

${ }^{13}$ Janz H. Die Behandlung von Eiterungen des Brustraumes mit Antibiotika unter Verwendung von Streptokinase-Streptodornase. Tuberkulosearzt 1952; 6: 25 - 28

${ }^{14}$ Jerjes-Sanchez C, Ramirez-Rivera A, Elizalde JJ, Delgado R, Cicero R, Ibarra-Perez C, Arroliga AC, Padua A, Portales A, Villareal A, Perez-Romo A. Intrapleural Fibrinolysis With Streptokinase as an Adjunctive Treatment in Hemothorax and Empyema. A Multicenter Trial. Chest 1996; 109: 1514 - 1519

15 Kärkölä P, Kairaluoma MI, Larmi TKI. Postpneumonectomy Empyema in Pulmonary Carcinoma Patients; Treatment with Antibiotic Irrigation and Closed-chest Drainage. J Thorac Cardiovasc Surg 1976; 72: $319-322$

${ }^{16}$ Laisaar T, Püttsepp E, Laisaar V. Early Administration of Intrapleural Streptokinase in the Treatment of Multiloculated Pleural Effusions and Pleural Empyemas. Thorac cardiovasc Surgeon 1996; 44: $252-256$

17 Landreneau RJ, Keenan RJ, Hazelrigg SR, Mack MJ, Naunheim KS. Thoracoscopy for Empyema and Hemothorax. Chest 1995; 109: $18-24$

${ }^{18}$ Liu HP, Chang CH, Lin PJ, Hsieh HC, Chang JP, Hsieh MJ. VideoAssisted Thoracic Surgery. The Chang Gung Experience. J Thorac Cardiovasc Surg 1994; 108: 834 - 840

${ }^{19}$ Mack MJ, Scruggs GR, Kelly KM, Shennib H, Landreneau RJ. Video-Assisted Thoracic Surgery: Has Technology Found its Place? Ann Thorac Surg 1997; 64: 211 - 215

${ }^{20}$ Mackinlay TAA, Lyons GA, Chimondeguy DJ, Barboza Piedras MA, Angaramo G, Emery J. VATS Debridement Versus Thoracotomy in the Treatment of Loculated Postpneumonia Empyema. Ann Thorac Surg 1996; 61: 1626 - 1630

${ }^{21}$ Moores DWO. Management of Acute Empyema. Chest 1992; 102: $1316-1317$ 
${ }^{22}$ Moulton JS, Benkert RE, Weisiger KH, Chambers JA. Treatment of Complicated Pleural Fluid Collections With Image-Guided Drainage and Intracavitary Urokinase. Chest 1995; 108: 1252 - 1259

${ }^{23}$ Neef H. Zur Behandlung des unspezifischen Pleuraempyems. Zbl Chir 1968; 18: 643 - 651

24 Pothula V, Krellenstein DJ. Early Aggressive Surgical Management of Parapneumonic Empyemas. Chest 1994; 105: 832 - 836

${ }^{25}$ Provan JL. The Management of Postpneumonectomy Empyema. J Thorac Cardiovasc Surg 1971; 61: 107 - 109

${ }^{26}$ Ridley PD, Braimbridge MV. Thoracoscopic Debridement and Pleural Irrigation in the Management of Empyema Thoracis. Ann Thorac Surg 1991; 51: $461-464$

${ }^{27}$ Robinson LA, Moulton AL, Fleming WH, Alonso A, Galbraith TA. Intrapleural Fibrinolytic Treatment of Multiloculated Thoracic Empyemas. Ann Thorac Surg 1994; 57: 803 - 814

${ }^{28}$ Sahn SA. Use of Fibrinolytic Agents in the Management of Complicated Parapneumonic Effusions and Empyemas. Thorax 1998; 53 (Suppl. 2): 565 - 572

${ }^{29}$ Schwemmle K, Mühe E, Krebs C. Die Therapie eitriger Erkrankungen der Lunge und des Pleuraraumes. Thoraxchirurgie 1974; 22: $420-424$

${ }^{30}$ Sendt W, Forster E, Hau T. Early Thoracoscopic Debridement and Drainage as Definite Treatment for Pleural Empyema. Eur J Surg 1995; 161: $73-76$

31 Silen ML, Weber TR. Thoracoscopic Debridement of Loculated Empyema Thoracis in Children. Ann Thorac Surg 1995; 59: $1166-1168$

${ }^{32}$ Smith JA, Mullerworth MH, Westlake GW, Tatoulis J. Empyema Thoracis: 14-Year Experience in a Teaching Center. Ann Thorac Surg 1991; 51: 39 - 42

${ }^{33}$ Strange C, Sahn SA. Management of Parapneumonic Pleural Effusions and Empyema. In: Wallace, RJ, ed. Infectious disease clinics of North America. Philadelphia: W. B. Saunders, 1991. $539-559$

${ }^{34}$ Strange C, Sahn SA. The Clinician Perspective on Parapneumonic Effusions and Empyema. Chest 1993; 103: 259 - 261

${ }^{35}$ Striffeler H, Ris HB, Würsten HU, Hof VI, Stirnemann P, Althaus U. Video-Assisted Thoracoscopic Treatment of Pleural Empyema. A new Therapeutic Approach. Eur J Cardiothorac Surg 1994; 8: $585-588$

${ }^{36}$ Striffeler H, Gugger M, Im Hof V, Cerny A, Furrer M, Ris H-B. Video-Assisted Thoracospic Surgery for Fibrinopurulent Pleural Empyema in 67 Patients. Ann Thorac Surg 1998; 65: 319 - 323

${ }^{37}$ Temes RT, Follis F, Kessler RM, Pett Jr SB, Wernly JA. Intrapleural Fibrinolytics in Management of Empyema Thoracis. Chest 1996; 110: $102-106$

${ }^{38}$ Tillet WS, Sherry S. The Effect in Patients of Streptococcal Fibrinolysin (Streptokinase) and Streptococcal Desoxyribonuclease on Fibrinous, Purulent and Sanguineous Pleural Exudations. Clin Invest 1949; 28: 173 - 190

${ }^{39}$ Ulmer JL, Choplin RH, Reed JC. Image-guided Catheter Drainage of the Infected Pleural Space. J Thorac Imaging 1991; 6: 65 - 73

${ }^{40}$ Wait MA, Sharma S, Hohn J, Nogare AD. A Randomized Trial of Empyema Therapy. Chest 1997; 111: 1548 - 1551

\section{J. M. Frey}

G. ö. Krankenhausgesellschaft Ibbenbüren $\mathrm{mbH}$ Abt. Thorax- und Kardiovascularchirurgie Große Straße 41 49477 Ibbenbüren 